\title{
LA GLOBALIZACIÓN Y SU IMPACTO EN ENFERMERÍA
}

\section{QUALITY OF WORKING LIFE IN THE NURSING STAFF: A REVIEW SYSTEMATIC IN THE CURRENT LITERATURA}

\author{
Recibido Junio \\ Aceptado Julio 2016
}

Correspondencia: Dra. Guadalupe Marcela Torres Escutia Canal de Ruppel Mz. 35 Lt. 19 Col. Insurgentes, Del. Iztapalapa, C.P. 09750.

\section{Autores:}

Dra. Guadalupe Marcela Torres Escutia Doctora en Ciencias de la Educación por el Colegio de Estudios de Posgrado de la Ciudad de México.

Maestra en Estudios Políticos y Sociales por la Facultad de Ciencias Políticas y Sociales de la UNAM.

Licenciada en Ciencias Políticas y Administración Pública por la Facultad de Ciencias Políticas y Sociales de la UNAM. Profesora de Ciencias Sociales en la Carrera de enfermería en la Facultad de Estudios Superiores Zaragoza de los módulos de: Enfermería Médico Quirúrgica l y II, Enfermería en el Proceso Reproductivo, Ecología Humana, Enfermería Comunitaria del 2005 al 2016.

Ing. Luis Antonio Rey Noeller Sandoval

Investigador y consultor independiente de minipymes y OSC's. Instructor y Diseñador de cursos certificado SEP/Conocer. Ex directivo de empresas Carrefour, IBM, American y Luqiid Gas.

Palabras clave: Globalización, Enfermería, salud y competencia.

Key words: Globalization, Nursing, Health and competition. 


\section{RESUMEN}

El siguiente trabajo tiene como finalidad reflexionar acerca del impacto que tiene el proceso de globalización en el personal de enfermería, concluyendo con la importancia que tiene el espíritu de emprendedor y la perspectiva de género.

Palabras claves: Globalización, Enfermería, salud y competencia.

Si bien la globalización surge de la dinámica del comercio y el mercado internacional que afecta directamente a las relaciones políticas y culturales así como en materia de tecnología donde el concepto vinculado a las TIC's (Tecnologías de la Información y Comunicación) y que impulsan un impresionante intercambio de información por medio del internet y los teléfonos móviles, herramientas dinámicas que transforman tiempo y espacio, y que impactan los procesos económicos, políticos, sociales, culturales, emocionales y espirituales; por esta situación los sistemas de salud no están al margen del ritmo de cambio y transformación que caracterizan el actual proceso de globalización.?

Dentro del modelo de "economía globalizada" el análisis y comparación de datos estadísticos permite revisar los procesos de participación e inserción productiva tanto de manera cuantitativa como cualitativa, así en términos estadísticos los indicadores per cápita y deciles de desarrollo humano, y en términos cualitativos las especialidades que exigen la evolución social, ambos transforman la perspectiva tradicional de problemas en áreas de oportunidad, y en un modelo de mejora continua.

En México desde el gobierno de Carlos Salinas de Gortari se impulso el Proceso de Reforma del Estado, que entre otras cosas implico la reducción del "tamaño del Estado", que no ha sido otra cosa, más que la venta de las empresas públicas, junto con la puesta en marcha de un proyecto poĺtico privatizador o neoliberal en donde impera la idea de que el acceso a los

\section{ABSTRACT}

The following paper aims to reflect on the impact of the globalization process nurses, concluding with the importance of the spirit of entrepreneurship and gender.

Keywords: Globalization, Nursing, Health and competition. servicios-beneficios sociales deben corresponder a una contraprestación por parte del individuo, sea ésta la realización de un trabajo o diedomente pagandoel servicio y por otro lado el hecho de que el gobierno no asigne mayor recurso o incluso lo reduzca, impacta en los centros hospitalarios y deja sin opciones al sistema, en una situación debancarrota donde uno de los grandes beneficiados en este proceso son los laboratorios farmacéuticos, quienes aprovechan el contexto del libre mercado para visualizar a la enfermedad como una industria de inversión y no como una industria de la salud, en el caso de la población tenemos aquellos que reciben un pésimo servicio por lo saturado que esta el sistema hospitalario: el escaso personal, la falta de medicamento y por el otro lado la población marginada que no tiene acceso a los servicios de salud, aspectos que se ven inmersos en la lógica del libre mercado.

Por esto es importante destacar a la Organización Mundial de la Salud ha definido a la salud como un estado de completo bienestar físico, mental y social, y no solamente la ausencia de afecciones o enfermedades; esta percepción es importante porque creemos que el ser humano está involucrado en diversos ámbito, que precisamente están vinculados a un concepto integral y ecológico que se tienen que considerar para lograr un equilibrio y es donde intervienen las diversas disciplinas para comprender el proceso salud-enfermedad en el individuo, como son: espiritual (humanismo), mental (psicología), cultural (antropología), social (sociología), laboral 
Rev. Enf. Neurol. (Mex)

(economía), entre otros.

Con la inquietud de enmarcar en un enfoque total el proceso salud enfermedad se hace necesario abordarlo desde el proceso de globalización que es una herramientas que nos hace considerar que hoy se vive en una aldea global y ahora lo que ocurra en el lugar más aislado, causa eco en todas las regiones del mundo.

Los acelerados cambios mundiales, nacionales, y locales determinan problemas para la salud importantes generando retos y al mismo tiempo ofrecen respuestas sociales efectivas para solucionarlos.

Villalobos señala que se ha ido construyendo toda una cultura de las competencias en educación a partir de la Declaración Mundial sobre la Educación Superior en el Siglo XXI de la UNESCO y del Proyecto de Turing. Esto tiene un fuerte impulso en el ámbito laboral y ha permeado lo que se conoce como el modelo de Educación Permanente. "Ésta busca formar a la persona para ofrecer respuestas que abarquen capacidades, aptitudes, talentos adecuados a los tiempos y exigencias de hoy, en el ámbito eminentemente empresarial." 2

De acuerdo a esto, se requiere formar a estudiantes en habilidades del pensamiento de orden superior e inferior, flexibles que les permita adaptarse al medio social y con conocimientos básicos generales que les permitan ser aplicados en cualquier tipo de trabajo.

En un universo de cambios acelerados y complejos los profesionales de enfermería están enfrentándose a desarrollar estrategias teórico-prácticas para entender e intervenir en los problemas de la salud.

Sin embargo, aunque existen estudios que muestran la imprescindible participación de los profesionales de enfermería, no se cuenta con la plataforma necesaria para incumir en los tan conocidos problemas de salud existentes. Además se afirma que los profesionales de enfermería tienen la capacidad de definir e identificar y tratar las demandas de salud de la sociedad con intervenciones efectivas en los escenarios de primer, segundo y tercer nivel de atención. Sin embargo, estos se ven limitados por las determinaciones de los actores políticos involucrados en la atención de la salud que no conocen la trascendencia de la misma, por ello es importante identificarlos para establecer la influencia que tienen para impulsar cambios en el ámbito de la Enfermería.

Los procesos económicos obliga a los gobiernos a vincular diversas temáticas como es el caso del trabajo, la educación y la salud, al respecto en octubre de 1998 en París la UNESCO organizo la Conferencia Mundial sobre Educación Superior donde se integraron las declaraciones y planes de acción producidos por las conferencias regionales que se habían realizado en el pasado y sirvieron de preparación, surgiendo como resultado una declaración mundial que marcarían las directrices, recomendaciones y transformaciones en el mundo solbre este tema. ${ }^{3}$

Debido a la influencia de lo anterior ya desde 2004 se profesionaliza la enfermería en México, que implica contribuir a dar un servicio de calidad permanentes, y es cuando las instituciones del sistema nacional de salud empezaron a contratar enfermeras con grado de licenciatura; esto ha implicado que con salario o plazas que en el pasado se contrataba a enfermeras técnicas, ahora se contrata a licenciadas, estas úllimas empiezan en el nivel más bajo del escalafón y en ese sentido las enfermeras técnicas han dejado de ser una opción de contratación, están desempleadas.

Al respecto, Vega en una nota periodística señala que José Arturo Ruiz, investigador del Instituto Nacional de Salud Pública (INSP), dice que el 52 \% de las enfermeras técnicas están desempleada o no trabajan en el área para la que estudiaron, situación a la que se le ha denominado "desperdicio laboral", ellas ocupan el segundo sitio en esta categoría después de los químicos fármaco-biólogos; a nivel licenciatura el desperdicio laboral es del 38\%."

\section{0} MMN Why Enf Neurol Vol. 15. No. 2 mayo - agosto 2016 
A pesar de esta situación, existe un importante déficit de personal de enfermería especializada en el sector salud, situación que impacta principalmente en hospitales de especialidad, como es el caso del hospital de Ciudad Victoria, Tamaulipas, o como el de Tláhuac, en el Distrito Federal, que no pueden operar a toda su capacidad por falta de ese recurso humano; es decir el sistema educativo forma enfermeras técnicas que no son contratadas, y por otro lado, aunque la matrícula de las licenciadas en enfermería que están egresando ha venido creciendo de manera importante, su número no es suficiente en términos per cápita para satisfacer la demanda del país. ${ }^{5}$

Al respecto el Plan Nacional de Desarrollo 2013-2018 establece que la labor del desarrollo y del crecimiento corresponde a: los actores, los sectores y todas las personas del país, además resalta el impulso que tiene que la sociedad se organice y contribuyan con su parte; para ello se establecen varias metas nacionales: un México en Paz, un México Incluyente, un México con Educación de Calidad, un México Próspero y un México con Responsabilidad Global.

La meta correspondiente a la Educación con Calidad señala que se necesita fortalecer el capital humano formar mujeres y hombres comprometidos con una sociedad más justa y más próspera que puedan tener las herramientas que potencialicen sus habilidades para interactuar con el sector empresarial y el social; es decir se debe establecer los vínculos efectivos entre escuela y trabajo; además de crear nuevas modalidades para que en el caso de enfermería, se generen nuevas formas de proporcionar el servicio. ${ }^{6}$

Como se puede observar existe un problema muy fuerte en la formación de capital humanos; la profesión de Enfermería es contemplada como parte del sistema de Educativo, por lo que las políticas al respecto tienen injerencia sobre la formación de las enfermeras.

La Organización Mundial de la Salud establece que la enfermería abarca la atención autónoma a personas de todas las edades, familias, grupos y comunidades, enfermos o no, y en todas circunstancias, además influye en la promoción de la salud, la prevención de enfermedades y la atención dispensada a enfermos, discapacitados y personas en situación terminal; es decir ellas son las protagonistas en los sistemas de salud ya que se les identifica como un grupo que tendrá cada vez mayor importancia en los temas de epidemiología y la población en general; son grupos vinculados fuertemente al desarrollo humano, porque los indicadores de salud son indicadores que son tomados en cuenta para tener la garantía de los avances de los países de primer mundo.

La profesión de Enfermería se desarrolla en tres niveles académicos: medio superior, licenciałura y posgrado; existe una extensa clasificación de las actividades que se realizan en Enfermería como son: la Asistencia Médica, Auxiliar de Enfermería, Auxiliar Medico, Enfermería Administrativa y Docencia, Enfermería Básica, Enfermería en Salud Publica/Atención Primaria, Enfermería Familiar, Enfermería General, Enfermería Homeópata, Enfermería Industrial, Enfermería Militar, Enfermería Pediátrica, Enfermería y Obstetricia, Enfermería Materno Infantil, Enfermería Medico Quirúrgica, Enfermería Militar y Enfermería en salud Mental/Psiquiátrica, Enfermería en el área de cuidados intensivos, Enfermería cardiovascular, Enfermería perinatal, Enfermería del Adulto en Plenitud, Enfermería en rehabilitación/Cultura física y deporte, Enfermería oncológica, Enfermería en otominolaringología?

Según datos del Anuario estadístico y geográfico de los Estados Unidos Mexicanos 2013, publicado por el INEGI señala que para el 2012, se contabilizaron dentro de las actividades vinculadas a Enfermería 326, 121 personal que realiza trabajo social, servicios auxiliares de diagnóstico y tratamiento, administrativas y otro personal dedicado a dicha actividad dentro del sector salud; Enfermeras dedicadas a otras labores se tiene registradas 31,844; Enfermeras especializadas se tiene un número de 29,382; las Enfermeras Generales, el sector salud tiene contabilizadas a 124,883; Enfermeras Auxiliares se tiene 90,824; es decir hay un total de

Enf Neurol Vol. 15. No. 2 mayo - agosto 2016 . 
603,053 enfermeras trabajando en los diversos espacios de acuerdo a datos arrojados por la Secretaria de Salud. ${ }^{8}$

De acuerdo a información de Juana Jiménez Sánchez quien es Directora de Enfermería y Coordinadora General de la Comisión Permanente de Enfermería de la Dirección General de Calidad y Educación en Salud en 2012 señaló que en el nivel licenciatura y técnico, cada año egresan en promedio 17,320 y 33,704 respectivamente, lo que genera una cifra anual aproximada de 51,024 egresos, además aproximadamente existen 723 escuelas de enfermería las cuales se clasifican en públicas o privadas, de estas 135 forman licenciadas y 588 técnicas ${ }^{9}$ como se puede observar el personal técnico casi dobla en cifras al personal de licenciatura y no va a ser contratado, e incluso el de licenciatura es subcontratado.

Como se puede observar estos procesos están obligando que el profesional de enfermería se adapte y enfrente a lo inmediato que es el desempleo - subemplearse, aspectos que demandan una modificación en la forma de pensar y de actuar.

En este sentido las y los enfermeros tienen que experimentar procesos radicales que causan ansiedad, conflicto y temor; es decir estos procesos implican pasar de estructuras rígidas de autoridad a procesos democratizadores; del individualismo al trabajo en equipo; de esperar la indicación del mando inmediato a la agilidad e inmediatez que implica la toma de decisiones; de esperar a que una institución te contrate a crear tu propio espacio laboral.

Estos procesos impulsan a asumir la responsabilidad que conlleva una acertada o errónea toma de decisiones; aprender a correr el riesgo del éxito y el fracaso; precisamente construir las líneas de la propia existencia o depositarlas a alguien ajeno a las aspiraciones de desarrollo del individuo; en este sentido las personas que han estudiado enfermería deben de empoderarse para hacer uso de herramientas que le permitan construir espacios laborales y de desarrollo.

Mujeres y hombres experimentan fuertes contradicciones, por ejemplo el grueso de las mujeres heredamos cargas culturales impresionantes como son: la obediencia al otro, el condicionamiento para la limpieza de los espacios y cuidado a los demás miembros de la familia, es decir la sociedad nos asigna los roles de madre-esposa; estos elementos impactan fuertemente en las enfermeras que refuerzan los aspectos de tipo cultural a la profesión, dándose lo que se ha llamado como la doble o triple jornada laboral pues son formadas bajo el marco de modelos educativos tradicional en donde se les fomenta la sumisión dependencia y respeto sin cuestionamiento a las jerarquías. En el caso de los hombres culturalmente se espera que sean fuertes, independientes, agresivos, activos, resistentes, que soporten el dolor y que sean ellos los violentos ${ }^{10}$; elementos que si se transformaran podrían generar nuevas relaciones de equidad, de respeto y podrían potencializar los trabajos con las propias compañeras.

Por todos estos aspectos es necesario potencializar al egresado de enfermería para que puedan desarrollar dos aspectos:

1. El espíritu de emprendedor para poder combatir el desempleo y fortalecer el desarrollo humano.

2. La perspectiva de género para construir liderazgos diferentes que desemboquen en el empoderamiento del personal que egresa de la carrera de enfermería.

\section{2} MMN Ent Neurol Vol. 15. No. 2 mayo - agosto 2016 


\section{REFERENCIAS BIBLIOGRÁFICAS}

1. Pacileo G. Globalización y tendencias actuales de la Salud Mundial en Globalización y Salud. Madrid: Ed. Federación de Asociaciones para la Defensa de la Sanidad Pública 2005; (consultado 1 de mayo de2015) P. 27-29 Disponible en: muw.nodo50.org/fadsp/pdf/globalizacion\%20y\%20salud.pdf

2. Villalobos Pérez, M. Evaluación de Aprendizaje basado en competencias. México: Ed. Minos; 2005. p. 22

3. Organización de las Naciones Unidas para la Educación, la Ciencia y la Cultura (UNESCO). Los dos primeros años del Decenio de las Naciones Unidas de la Educación para el Desarrollo Sostenible (2005-2014) París, Francia 2007. (Consultado: 22 de marzo 2015) Disponible en: unesdoc.unesco.org/ images/0015/001540/154093s.pdf

4. Vega Margarita. Pega desempleo a las Enfermeras. Alerta especialista sobre bajos salarios. Llaman a revisar la formación de personal con nivel técnico. Periódico Reforma 6 de enero 2012. Disponible en: panoramalaboral-conampros.blogspot. com/2012/.../panorama-laboral-06-07-2012.html

5. Alatorre, A. Hospitales de especialidad sufren crisis de enfermeras. Portal de la Secretaria de Salud. 05 de enero del 2013.(consultado 7 de abril de 2015) Disponible en: https://noticias.terra.com.mx/mexico/hospitales-de-especialidad-sufren-crisis-de-enfermeras,e3e26342dab0c310VgnVCM20000099ccebOaRCRD.htmi
6. Secretaría de Gobernación Plan Nacional de Desarrollo 2013-2018. México 2013. Disponible en: pnd.gob.mx/

7. Instituto Nacional de Estadística Geografía e Informática (INEGI) Catálogo de Codificación de Carreras, censo de población y vivienda México 2005 (consultado 14 de noviembre 2015) pp. 28-29,74-75 Disponible en: www.inegi org.mx/est/contenidos/espanol/metodologias/enoe/.../carreras.pdf

8. Instituto Nacional de Estadística Geografía e Informática (INEGI) Anuario es tadístico y geográfico de los Estados Unidos mexicanos. México; 2015. (consultado 16 de mayo de 2015) disponible en: http://www3.inegi.org.mx/sistemas/componentes/previsualizador/vista.aspx?arch=/prod_serv/contenidos/ espanol/bvinegi/productos/nueva_estruc/aegeum/2015/702825077280 pdf\&tipo=1

9. Jiménez, Sánchez Juana. Formación de Recursos Humanos en Enfermería. La Calidad de la Atención a la Salud en México. México: SEP.; 2012. (consultado: 27 de agosto de 2015) pp 191 Disponible en: www.calidad.salud.gob.mx/ site/editorial/.../dgr-editorial_01M.pdf

10. Lagarde y de los Ríos, M. Los cautiverios de las mujeres: Madresposas, Monjas, Presas, Putas y Locas. 2da. ed. México: UNAM; 2011.

\section{FE DE ERRATAS}

En la edición anterior Volumen. 15. No.1 enero - abril 2016 de la Revista de Enfermería Neurológica ha aparecido un error en los autores en el siguiente artículo: " La subjetividad del fenómeno, efectos adversos alrededor de los cuidados de enfermería".

ERROR: Página 10, En el Párrafo de autores.

DICE: Solis Matamoros Elizabeth, Amador Meneses Sandra, Aizpuru-Sulis Libe, Camarillo Sánchez Karla P., Reyes López Magali, Liliana Mendoza Martínez, Landeros Olvera Erick.

DEBE DECIR: Solis Matamoros Elizabeth, Amador Meneses Sandra, Aizpuru-Sulis Libe, Camarillo Sánchez Karla P., Reyes López Magali, Mendoza Martínez Liliana, Najera Gutiérrez Gudelia, Landeros-Olvera Erick. 